\title{
Digital Model Construction and Precision Structural Analysis Of Bridges Based On Full Field and Life Circle Intensive Measurement
}

\author{
YUAN Xiang Rong \\ School of Civil Engineering, Guangzhou University, Guangzhou 510006, Guangdong, China \\ rongxyuan@163.com
}

\begin{abstract}
Keywords: Digital structure model, digital design model, digital calculation model, digital measurement model, digital test model
\end{abstract}

Abstract. Two aspects are needed to improve in the research and practice of current bridge structural test. First, test data is incomplete compare with calculation data, and survey times are insufficient compare with the bridge life circle. Second, although the analysis and calculation accuracy can not be always guarantee, the test program is formulated based on the results of structural analysis and calculation, and the assessment of the test results is determined by the comparison of the structural calculation results and the measured results. Therefore, digital image methods are suggested here for the full-field and spatially intensive measurement of the bridge. And the digital test structural models and the parametric test models which are isomorphic with the calculation model are suggested to construct based on the full-field data. Consistent with the stages of the structure life circle, multiple generations of digital design model, digital calculation model, digital measurement model and digital test model are established through intensive inspection, testing and identification. The 4 types of digital models are integrated in digital model set. The parameters and outputs of the set are precisely analyzed. Digital consistency and convergence of the parameters and output results of the sets of all generations are studied. Current state and previous history of the bridge structure is graphically and digitally represented by the comparison results of the contemporary digital model set with each generation of digital models set. The future state and static or dynamic load test of the bridge structure are virtue described and performed. The virtue test results are used to construct the virtue digital model sets of the bridge structure. The structure aging process is recorded and expected, the structure load capacity is digitally expressed, and the structure remaining life is estimated through the precisely analysis of the property characteristics, track changes, trends and development process of the various parameters and the response of the digital model sets.

\section{Introduction}

Under the present situation of bridge engineering, structure design is digital described as CAD diagram, and the structural analysis is digital expressed by the finite element method. But the digitized decree of bridge test is far behind that of design or calculation. At least three main deficiencies of the bridge test are needed to improve.

First, measurement data is incomplete. Data type is incomplete, coverage of the test bridge is incomplete, time coverage is incomplete with respect of the bridge life circle, and load process of the bridge is incomplete. The bridge design is a complete set of geometry, physics and mechanics. The bridge calculation response is a complete set of internal force and deformation data. But the measure mesh of the bridge is very rough compare with the FEM mesh; the bridge test time interval is rather long, the short is 1,2 years, the length is more than ten years.

Second, the calculation data is unreliable. The results of FEM calculation of large complex structures are usually required to be verified, but the state of the service bridges are assessed through the comparison of the results between the test and calculation. According to the current technical code of inspection and evaluation for bridges, the state of the bridge is assessed by the results comparison between the test response value and design value. But the design value is often missing, and is replaced by the calculation value. Questioned calculation value is used to assess the state of unknown structure, certainty and accuracy results are in question. 
Third, there is no digital test model. There is CAD model for the bridge design, which can be called as digital design model (DDM). The bridge can be described in detail (such as the construction diagram). There is FEM model for the bridge calculation, which can be called as digital calculation model (DCM). The bridge can be described by the static and dynamic characteristics of the structure, and can be calculated by a large number of loading modes. There is measure data in bridge test, (often incomplete), but there is no digital test model. Limited test data means rough description of the structure and limited state, such as load is enough or not enough, work performance is good or bad, elastic or plastic.

Therefore, it is necessary to focus on the research of the test method of full field, life circle, intensive and completeness, the construction of Digital Measurement Model (DMM), and Digital Test Model (DTM) based on above test method, the relationship of the measurement and test models with the design and calculation models, and the structural precision analysis based on above 4 digital models. It is significant in the description, track change and trend forecast of the bridge health, and the operation, management and future planning and design of the bridge. For the large and complex structures such as long-span suspension bridge, cable stayed bridge and combined arch bridge, it is difficult to measure the structure of whole field and intensive. But technical progress can be expected, economic problem can be overcome for the simple beam bridges, and the technology application prospect in large complex structure can be anticipated.

\section{Significance of the Digital Bridge Model}

The DDM of CAD diagram of the bridge is designed according to certain requirements. The structural behavior is analyzed through DCM of FEM. The real bridge constructed according to the DDM of the construction diagram must be inspected to verify the degree of compliance with the preset target. In general, the inspection results are compared with the results of DCM. In the whole life cycle of the bridge, structure heath is evaluated in this same way. For example, according the inspection code of highway and city bridges [1-3], in load test evaluation, the response ratio of test load to design load is the ratio of test loading effect calculation value and the design loading effect calculation value. Test scheme is designed according this ratio. The structural verification coefficient is the ratio of the measured value and the corresponding theoretical calculation value, the working performance and safety reserve of the bridge is judged by the verification of calibration.

Firstly, the main basis of the test scheme (designed according the response ratio of test load to design load) and the test conclusion (determined according the verification coefficient) is the results of the analysis and calculation of the structure. DCM has the completeness advantages of model parameters and response data, but its accuracy is questionable. For example, in the analysis and calculation of a long span composite arch bridge, the independent calculation results of several researchers are difficult to keep consistent. Moreover, in many cases, the original design of the bridge can not be found, and the structure design model must be reconstructed based on the field measurement. According to the calculation results, the reliability should be carefully treated. According to the results of calculation and test, the reliability of the structure assessment should be treated with caution. Therefore, it is necessary to establish digital test model which is isomorphic to calculation models in the engineering structure scale to ensure the correctness of the calculation.

Second, the test results have the advantages of data reliability and accuracy, but they are far from completeness. At present bridge load test, only $2 \sim 4$ cross sections of the beam are inspected. The calculation results are compared with the experiment results at these sections. The great majority of the whole field, all typed, detailed, and comprehensive calculation data is very unfortunately discarded. Therefore, it is necessary to study and develop a whole field and intensive measure method to obtain the test data field of space isomorphism with the finite element solution.

Third, in present bridge load test, the experience is reliable, and the calculation is approximated guaranteed, test result is usable. But the description of the structure is qualitative rather than quantitative. When structural deformation and internal force changes in linear elastic range, the bridge 
can be considered working in good property. When the calibration coefficient meet with the requirements, the load capacity of the structure can be considered as enough. Conclusion is binary (good or bad) or rough. Although the conclusion is basically reliable and usable, but the precision and fine judgment of the bearing capacity and the remaining life can not obtained by the conclusion. Therefore, it is necessary to realize the test analysis as accurate and detailed as FEM analysis in the engineering structure scale.

Fourth, in the operating life of the bridge, its status is gradually change, inspection interval of several years even ten years is not enough to monitor the time history of the structure health state. The comparison of each test data is scarce, and the health trend is difficult to describe. So it is necessary to narrow the time interval of test, and to do precision and fine analysis of the digital model of the bridge in time and space.

\section{Digital Measure model and Digital Test Model}

Design and calculation have their own digital model, and the test model should be constructed based the inspection data. Through measuring, testing and analyzing the measurement data, the initial Digital Measure Model DMM0 is constructed corresponding to the Digital Design Model DDM, and the initial Digital Test Model DTM0 is constructed corresponding to the Digital Calculate Model DCM. DDM, DCM, DMM0 and DTM0 are integrated in the initial reference Digital Structure Model Set DSMS0. The initial model set is considered as the benchmark set.

The change of the geometric, physic and mechanic parameters of structure caused by structure maintain and strength is recorded in $\operatorname{DDM}(\mathrm{i})(\mathrm{i} \in \mathrm{Z})$. The difference between the real structure and the design structure found in structure inspection, measurement and test is recorded in $\operatorname{DMM}(j)(j \in Z)$. The new calculation model DCM $(\mathrm{k})(\mathrm{k} \in \mathrm{Z})$ is constructed according to the changed DDM and DMM. The new test model DTM(m) $(\mathrm{m} \in \mathrm{Z})$ is constructed in turn after each structure load test. The four models can be compared with each other, can be compared with the benchmark model, and can be compared with each generation. $\operatorname{DDM}(\mathrm{i}), \operatorname{DCM}(\mathrm{k}), \operatorname{DMM}(\mathrm{j})$ and $\operatorname{DTM}(\mathrm{m})$ are integrated in the nth generation Digital Structure Model Set DSMS(n).

Through the comparison of each generation set DSMS(n) and the benchmark set DSMS0, the corresponding state of the bridge can be assessed. Through the digital structure model set DSMS compared with each other, the change path of the bridge state can be clearly described, and the structure's load capacity and the remaining life can be determined and forecast. The consistence and harmony of the output data of the DSMS are studied on the data types and space distribution. The change, develop, and trend of the structure state can be revealed.

DMM established by the structure measurement would be isomorphic with DDM. The graphic description of the DDM is similar as that of CAD diagram. DTM established by the structure load test would be isomorphic with DCM. The displacement measure mesh is corresponding to the calculation mesh.

Generally, precision of digital image detection is 0.01 pixels[4], the resolution of digital camera is higher than 80 million pixels $(8000 \times 10000)$. When the coverage area of the image is a 100 meters long, 1 pixel represents 1 centimeter, and 0.1 millimeter precision can be obtained. Structure measurement precision by image detection is as good as traditional measure technology. In the structural parameters identification or element identification (Amendment), the relative error between the test and the analysis data is less than $1 \%[5]$.

Image Measure Finite Element method should be established based on the digital image deformation detection and element parameter identification. The image deformation detection mesh is consistent with that of FEM method, the shape function of the FEM is used to identify the element parameter based on the image detection. Finite Element Image Measure Method should be studied based on the 
digital image correlation techniques, and the image deformation is represented as the shape function of the FEM.

DTM established by the complete data field can be used to analyze the structure similar as FEM.

The virtue bridge test in situ can be operated through the DTM.

3D virtue environment[6] should be built corresponding to the DDM, DCM, DMM and DTM. The geometrical shape and deformation of the structure can be expressed graphically. The displacement and stress of the structure element can be displayed in the form of a pop - up window.

The scrip of the bridge load test can be edited, the virtue bridge test can be deduced according to the script, and the process of the test can be interfered by the input device. The pre fabricated test scheme can be directly (or slightly modified) for the actual bridge inspection or static load test. The basic data in the virtual test report is basically consistent with the data in the field measurement report. The abnormal data can be predicted and analyzed, and the inconsistent data can be judged and explained.

\section{Summary}

1. The significance of the digital bridge structure model is introduced.

2. Image Measure Finite Element method and Finite Element Measure Image method are suggested to acquire the whole field and intensive test data, and to identify the element parameter of the structure. 3. The digital measure model and digital test model are suggested to integrate into digital structure model set with the digital design and calculation model.

4. The function of the digital structure model is described.

\section{Acknowledgements}

This work was financially supported by the National Natural Science Foundation (51278137), and the Guangdong Provincial Science and Technology Project (12C42011564).

\section{References}

[1] The People's Republic of China Industry Recommended Standards, Specification for Inspection and Evaluation of Load-bearing Capacity of Highway Bridges, China Communication Press, Beijing, 2011

[2] Guangdong Province Code, Technical Standard for Inspection of Urban Bridge, China Building Press, Beijing, 2012

[3] The People's Republic of China Industry Recommended Standards, Technical Code of Inspection and Evaluation for City Bridges (proposed code), http://www.risn.org.cn/News/ShowInfo.aspx, 2013

[4] L. B. Meng, Study and Application of the Digital Spackle Correlation Method, PhD Thesis, Tsinghua University, 2005

[5] X. R. Yuan, Step update method for the large parameter error model, J. Shijiazhuang Railway Institute, 2 (1996) 19-25

[6] X. R. Yuan, The basis and significance of structure virtual and actual construction technology research, Guangzhou Building. 6 (2014) 3-9 